\title{
Epithelial hypoxia-inducible factor- 1 is protective in murine experimental colitis
}

\author{
Jörn Karhausen, ${ }^{1}$ Glenn T. Furuta, ${ }^{1,2}$ John E. Tomaszewski, ${ }^{3}$ Randall S. Johnson, ${ }^{4}$ \\ Sean P. Colgan, ${ }^{1}$ and Volker H. Haase ${ }^{5}$

\begin{abstract}
${ }^{1}$ Center for Experimental Therapeutics and Reperfusion Injury, Brigham and Women's Hospital, Boston, Massachusetts, USA. ${ }^{2}$ Combined Program for Pediatric Gastroenterology and Nutrition, Children's Hospital, Boston, Massachusetts, USA. ${ }^{3}$ Department of Pathology, University of Pennsylvania School of Medicine, Philadelphia, Pennsylvania, USA. ${ }^{4}$ Division of Biology, University of California at San Diego, La Jolla, California, USA. 5Department of Medicine, University of Pennsylvania School of Medicine, Philadelphia, Pennsylvania, USA.
\end{abstract}

\begin{abstract}
Mucosal epithelial cells are uniquely equipped to maintain barrier function even under adverse conditions. Previous studies have implicated hypoxia in mucosal tissue damage resulting from both acute and chronic inflammation. Given the importance of the transcriptional regulator hypoxia-inducible factor-1 (HIF-1) for adaptive hypoxia responses, we hypothesized that HIF-1 may serve as a barrier-protective element during mucosal inflammation. Initial studies of hapten-based murine colitis revealed extensive mucosal hypoxia and concomitant HIF-1 activation during colitis. To study this in more detail, we generated 2 mouse lines with intestinal epithelium-targeted expression of either mutant Hifla (inability to form HIF-1) or mutant von Hippel-Lindau gene ( $\mathrm{Vblh}$; constitutively active HIF-1). Studies of colitis in these mice revealed that decreased HIF-1 expression correlated with more severe clinical symptoms (mortality, weight loss, colon length), while increased HIF levels were protective in these parameters. Furthermore, colons with constitutive activation of HIF displayed increased expression levels of HIF-1-regulated barrier-protective genes (multidrug resistance gene-1, intestinal trefoil factor, CD73), resulting in attenuated loss of barrier during colitis in vivo. Taken together, these studies provide insight into tissue microenvironmental changes during model inflammatory bowel disease and identify HIF-1 as a critical factor for barrier protection during mucosal insult.
\end{abstract}

\section{Introduction}

Colonic epithelial cells are anatomically positioned to provide a selective barrier to luminal antigens and pathogens. Supported by a complex vasculature, this physiologically crucial barrier is a primary target for diminished blood flow and resultant tissue hypoxia. A number of studies suggest a close association of inflammation and hypoxia at the tissue level. In Crohn disease, a chronic intestinal inflammatory process that is, at least in part, characterized by marked abnormalities of gut barrier function, microvascular disturbances have been consistently demonstrated (1-3). Consequently, observations from both human disease $(4,5)$ and experimental colitis $(6,7)$ suggest the presence of colonic hypoxia and its significance in the disease process. Interestingly, a number of barrier-protective genes are critically regulated by hypoxia-inducible factor-1 (HIF-1), including intestinal trefoil factor (ITF), CD73, and multidrug resistance gene-1 (MDR1) (8-10). Among these, ITF and CD73 appear to serve as barrier-protective elements during hypoxia in vivo (9, 10). Moreover, Mdr1- and Itf-null mice display either spontaneous development of colitis (Mdr1-null) (11) or increased susceptibility to chemically induced colitis (Itf-null) (12).

HIF-1 is a member of the Per-ARNT-Sim domain family (also referred to as PAS domain family) of basic helix-loop-helix transcription factors. As a global regulator of oxygen homeostasis, this

Nonstandard abbreviations used: EF5, 2-[2-nitro- $1 H$-imidazol-1yl]-N-[2,2,3,3,-pentafluoropropyl] acetamide; Fabp, fatty acid-binding protein; GLUT-1, glucose transporter-1; HIF, hypoxia-inducible factor; ITF, intestinal trefoil factor; MDR1, multidrug resistance gene-1; P-GP, P-glycoprotein; TNBS, 2,4,6-trinitrobenzene sulfonic acid; VHL, von Hippel-Lindau.

Conflict of interest: The authors have declared that no conflict of interest exists.

Citation for this article: J. Clin. Invest. 114:1098-1106 (2004).

doi:10.1172/JCI200421086. $\alpha \beta$ heterodimeric transcription factor facilitates both oxygen delivery and adaptation to oxygen deprivation (13). Activation is dependent on stabilization of the oxygen-sensitive $\alpha$ subunit and subsequent nuclear translocation to form a functional complex with HIF-1 $\beta$ and cofactors such as CBP and its ortholog p300 (recently reviewed in ref. 14). In conditions of adequate oxygen supply, ironand oxygen-dependent prolyl-hydoxylation of the oxygen-dependent degradation domain within HIF- $1 \alpha$ is necessary for binding to the von Hippel-Lindau (VHL) tumor suppressor protein (pVHL) and consequently to the E3 ubiquitin ligase complex, which targets it to proteasomal degradation $(15,16)$. Absence of functional pVHL therefore results in constitutively active HIF-1 $\alpha$, an effect that we have used to overexpress HIF-1 experimentally. A second hypoxic switch operates in the carboxy-terminal transactivation domain of HIF-1 $\alpha$ with the hydroxylation of an asparagine residue; in hypoxia, asparagine hydroxylation is blocked and CBP/ p300 recruitment is facilitated (17).

Initial observations have indicated that colonic epithelia become severely oxygen-deprived during inflammation, and that HIF-1 activation and function are apparent in vivo. In the current study we therefore aimed to clarify whether HIF-1 activation and function are central to innate protective mechanisms during model inflammatory disease (colitis). In order to assess the pathophysiologic relevance of HIF-1-regulated gene expression in this disease process, we used Cre-loxP-mediated recombination to delete Hifla or Vhlh (gene symbols for murine HIF-1 $\alpha$ and $V H L$ ) in intestinal epithelia, which resulted in either absent or constitutively active HIF-1 within this tissue. Both in whole-body hypoxia and in model colitis, the importance of HIF-1 expression for the intestinal barrier integrity was empirically demonstrated, with the levels of HIF-1 expression correlating with protection from 2,4,6-trinitrobenzene 

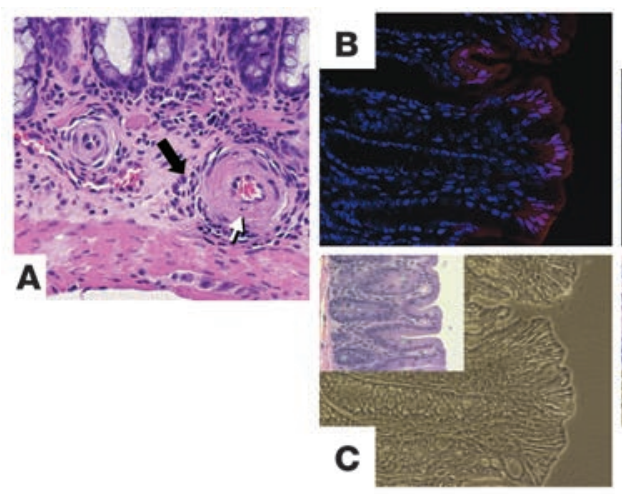

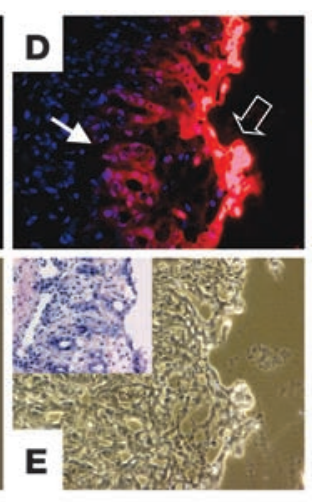

H

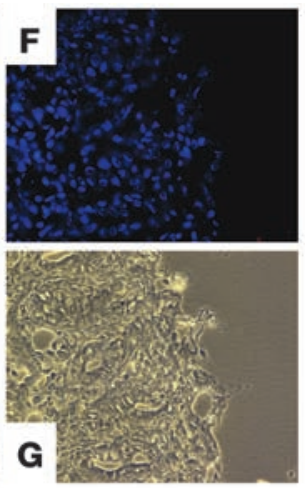

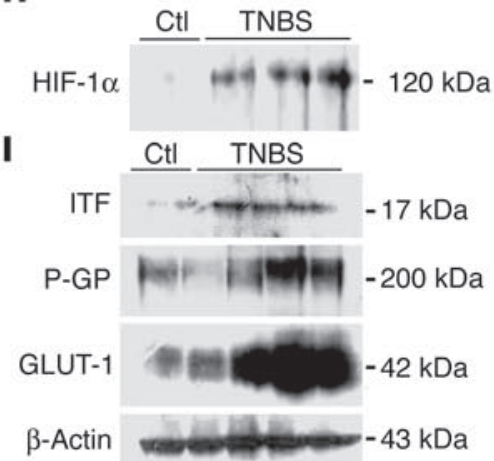

\section{Figure 1}

Epithelial hypoxia in TNBS colitis is associated with inflammatory lesions. (A) H\&E staining in TNBS colitis (7 days after induction). In an area of relatively minor epithelial inflammation, submucosal vessels are encircled by a mixed population of inflammatory cells (filled arrow). Fibrinoid necrosis of the vessel walls (open arrow, with apoptotic bodies) and signs of luminal obliteration are observed. Magnification, $\times 600$. (B) Localization of EF5 (nuclear counterstaining with DAPI) in a colonic section from a vehicle-control animal at day 3 . Discrete immunofluorescence in surface epithelial cells and underlying crypts. (C) Corresponding phase-contrast image. Inset: H\&E stain of an adjacent section. (D and E) Section taken from distal colon in TNBS-exposed animals. (D) Intense EF5 immunofluorescence overlying the area of ulceration (open arrow) and in underlying epithelial portions (filled arrow). (E) Phase contrast of $\mathbf{D}$. Inset: H\&E staining of an adjacent section from the same tissue, displaying erosion of the epithelial layer and beginning inflammatory infiltration. (F) Competed stain (section adjacent to that shown in $\mathbf{D}$ and $\mathbf{E}$ ). Antibody specificity is documented by absence of Cy3 signal when antibody was saturated with free drug. (G) Corresponding phase-contrast image. Magnification in B-G, $\times 400$. (H) HIF-1 $\alpha$ stabilization in TNBS colitis. Western blot analysis from TNBS and vehicle-control (Ctl) animals 7 days after induction of colitis. Blots are derived from nuclear extracts from the colon of control and TNBS-treated animals. (I) Western blot analysis of the HIF-1-responsive genes ITF, P-GP, and GLUT-1 in control and TNBS-treated animals.

sulfonic acid-induced (TNBS-induced) colitis in a number of clinical parameters (i.e., weight loss, colon length, intestinal permeability). These findings indicate the presence of an orchestrated induction of barrier-protective genes in the colon, with epithelial HIF-1 expression being essential for barrier integrity. Results from these studies suggest that HIF-1 expression is associated with clinical benefit for disease outcome and might constitute a novel therapeutic approach for inflammatory diseases associated with significant hypoxia, such as the inflammatory bowel diseases.

\section{Results}

"Physiologic hypoxia" of the colonic epithelium is greatly enhanced in colitic lesions. Initially we sought to consolidate previous reports $(2,5-7)$ implicating hypoxia in mucosal inflammatory diseases such as inflammatory bowel disease. For this purpose, we used colitis as a model of mucosal inflammation, and since previous observations (6) suggest that TNBS colitis best approximates the functional and anatomical microvascular abnormalities that have been observed in human Crohn disease patients (4), we initially used the TNBS colitis model. Close histological examination of colonic tissue derived from TNBS-colitic animals ( 7 days after induction) revealed a characteristically obliterating vasculitis of the small submucosal vessels in loose association with mucosal inflammation (Figure 1A), which is similar to observations in human specimens $(5,18)$.

To determine the extent of tissue hypoxia in this experimental model, we utilized the characteristic reduction and binding of the nitroimidazole compound 2-[2-nitro- $1 \mathrm{H}$-imidazol-1yl]-N-[2,2,3,3,pentafluoropropyl] acetamide (EF5) to cellular macromolecules in the absence of adequate oxygen levels (19). As shown in Figure 1B, colonic samples from vehicle-treated control animals revealed evident EF5 retention in superficial epithelial layers within the colon, consistent with reports suggesting that the intestine is relatively hypoxic compared with other mucosal tissues (20). In agreement with recent observations examining EF5 localization in the small intestine (10), such EF5 staining was almost exclusively localized to the epithelium. In contrast, animals exposed to the colitis-inducing hapten TNBS revealed a profound retention of EF5 within colonic epithelia (Figure 1D). Moreover, such EF5 retention was prominently associated with colitic lesions both in superficial and in deeper submucosal regions of the mucosa (compare insets in Figure 1). Such findings indicate that the TNBS-induced vascularity changes likely result in significant tissue hypoxia, predominantly within the epithelium.

HIF is a central regulatory transcription factor for hypoxiainduced gene expression and serves as a sensitive and selective indicator of hypoxia (13). Based on our findings with EF5 localization, we hypothesized that TNBS colitis results in HIF-1 activation, particularly within the epithelium. Since regulation of HIF-1 occurs predominantly through stabilization of the $\alpha$ subunit in response to hypoxia, we analyzed HIF-1 $\alpha$ levels in colonic tissue extracts derived from TNBS and vehicle-control mice by Western blot. As can be seen in Figure 1H, TNBS colitis resulted in prominent HIF-1 $\alpha$ expression compared with vehicle control. Such HIF-1 $\alpha$ expression was evident by day 1 after TNBS (data not shown) and was most significant compared with that in controls at day 7 . We extended these findings to determine whether HIF-1-regulated gene products were also induced in TNBS colitis. At day 7 after induction, both the wellcharacterized HIF-1-responsive gene product glucose transporter-1 (GLUT-1) (21) and 2 more recently characterized barrier-protective gene products, ITF and the MDR1 gene product P-glycoprotein (P-GP) $(8,9)$, were prominently induced (Figure 1I). These findings reveal correlative expression of HIF-1-regulated genes at time points at which significant hypoxia and HIF-1 activation are evident.

Generation and characterization of conditional Hifla- and Vhlh-deficient mice. To better define the role of HIF-1 in colitis, we generated mice that either lack or exhibit constitutive high levels of Hif-1 $\alpha$ in colonic epithelia by Cre-loxP-mediated recombination. To achieve 

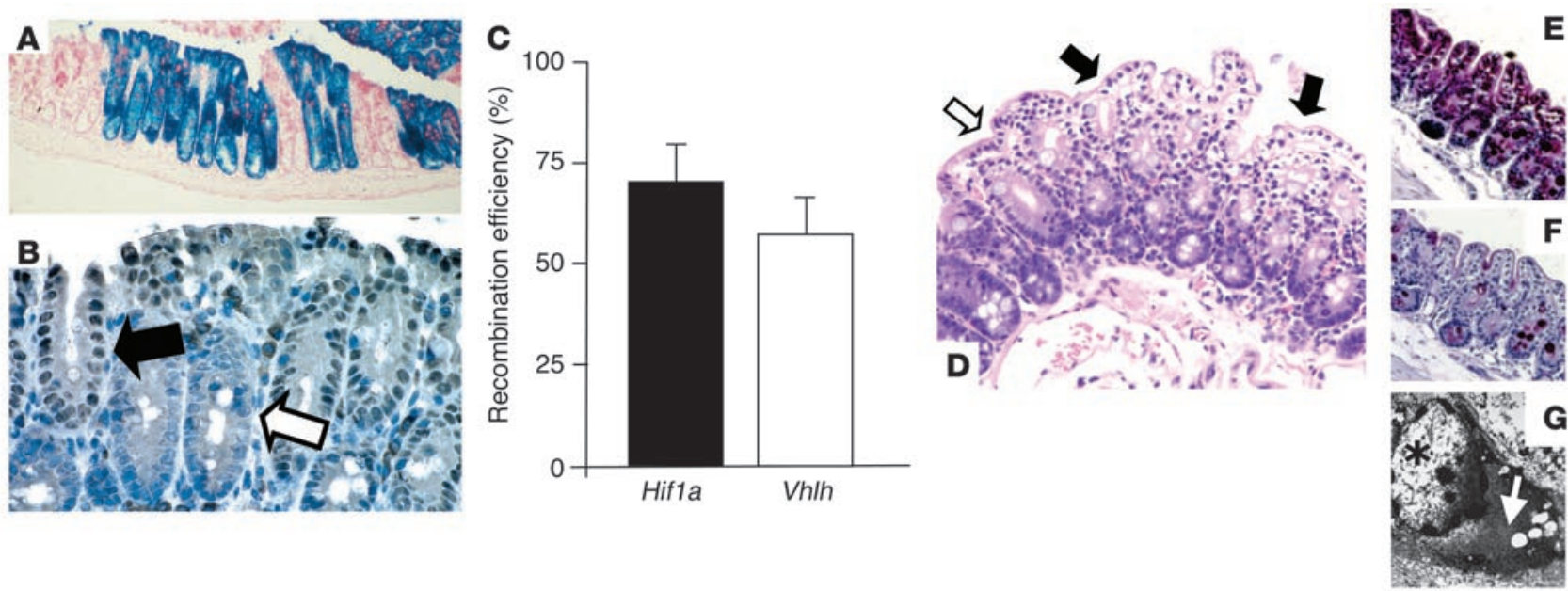

\section{Figure 2}

Fabp-Cre-mediated recombination in the colon: analysis of Fabp-Cre activity and histological characterization of mutant mice. (A and B) FabpCre activity as determined by a lac $Z$ reporter transgene and immunohistochemical staining for Cre-recombinase. (A) Section of the cecum stained with X-gal. Recombination occurs always in all cells of an individual crypt. Magnification, $\times 100$. (B) Paraffin-embedded section of the colon from a Vhlh mutant mouse. Consistent with the recombination process in the lacZ transgenic mice, all cells within a Cre-positive crypt display nuclear-localized Cre signal (filled arrow), while all cells in a negative crypt stain negatively (open arrow). Magnification, $\times 400$. (C) Determination of the efficiency of recombination by quantitative PCR. Levels of expression of the recombined gene (Hif1a or Vhlh) were compared with the expression levels of a non-recombining control gene in genomic DNA from isolated colonic epithelial cells ( $n=3$ for each genotype). (D-G) Clear-cell changes in the Vhlh mutant colon. (D) Luminal epithelial cells with pronounced clearing are indicated by filled arrows; the open arrow points to adjacent luminal cells without clearing. Clearing is a result of glycogen accumulation in Vhlh mutant cells, as shown by diastasesensitive PAS staining. (E) PAS stain without diastase treatment. (F) PAS stain after diastase treatment of the adjacent colon. (G) Ultrastructural analysis of clear cells showed accumulation of cytoplasmic material that is morphologically consistent with glycogen (white arrow). The star indicates the nucleus of a clear cell. Magnification in D-F, $\times 200$.

efficient gene deletion targeted to the colon, we used mice that express Cre-recombinase under transcriptional control of the fatty

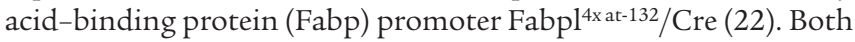
lac $Z$ reporter gene expression (Figure $2 \mathrm{~A}$ ) and Cre expression (Figure $2 \mathrm{~B}$ ) indicate that recombination occurred in all cells within the crypt, which suggests that Cre-mediated recombination in Hifla and Vhlb conditional mutants is initiated early, presumably in crypt progenitor cells. Consistent findings have been previously reported using the Fabp promoter (22). Furthermore, in accordance with those findings is the complete recombination of either target gene in transitional epithelial cells of the bladder, urethra, and renal pelvis. Recombination in other organs such as brain, liver, heart, lung, and muscle was not detected by Southern blot analysis or with a lacZ reporter transgene (23), nor did we observe recombination in the germ line (data not shown). Recombination efficiency for Hif1a and Vhlb 2-lox alleles was quantified by realtime PCR of genomic DNA isolated from epithelial-enriched colon samples, parallel to the method described by Cramer et al. (24). The Hif1 a 2-lox allele recombined with an efficiency of $72 \% \pm 8 \%$, while Cre-mediated recombination for the Vhlb 2-lox allele was slightly less $(60 \% \pm 5 \%$; Figure $2 \mathrm{C})$. Recombination efficiency within the different areas of the colon (i.e., cecum vs. colon) was consistent (data not shown). Both conditional mutants (Hifla ${ }^{2 l o x / 2 l o x} ; \mathrm{Fabp}^{4 \mathrm{xat}-}$ $132 / \mathrm{Cre}$ and $V h l h^{2 l o x} / 2 l o x ; \mathrm{Fabpl}^{4 x}$ at-132/Cre) appeared healthy without overt phenotypic differences, were fertile, and had a normal lifespan. Careful macroscopic examination of both Fabp-Cre mutants showed a smooth colonic mucosa without evidence of polyps, ulcerations, or vascular malformations. Bladder, urethra, and renal pelvis also appeared normal. While histological examination of
Hif1 a mutant colon showed no aberrances, all Vhlh mutant colons, but none of the control colons, displayed patches of cytoplasmic clearing, which was most pronounced toward the villus tip (Figure 2D). Less pronounced clearing was furthermore found in transitional epithelial cells of the bladder (data not shown). Clearing of cells is generally a result of glycogen and/or lipid accumulation and has been shown to be associated with Vhlh deficiency in other cell types (25-27). Here, we found that clearing in mutant colonic epithelium is mainly a result of glycogen accumulation, as shown by a diastase-sensitive PAS stain (Figure 2, E and F) and electron microscopy (Figure 2G). Furthermore, as pVhl also functions as a tumor suppressor gene, we examined Vhlh mutant colons for abnormal rates of proliferation. Careful histological examination and staining with the proliferation marker Ki-67 (data not shown) did not display any difference between clear cell-positive and clear cell-negative colon crypts in mutant animals, nor did we observe differences between mutants and control mice. These findings suggest that $\mathrm{pVhl}$ deficiency per se does not lead to abnormalities in epithelial cell growth regulation in the murine colon.

Conditional deletion of Hifla in epithelia worsens the clinical course of colitis. We first determined whether induction of HIF- $1 \alpha$ protein during whole-body hypoxia (Figure 3A) or in the setting of TNBS-induced colitis (Figure 3B) was significantly altered in Hifla mutants when compared to WT littermates. Despite recombination efficiency below that reported using the Cre-loxP strategy (24), colonic epithelial-enriched samples derived from conditional mutant colons lacked the induction of HIF-1 $\alpha$ protein that was apparent in littermate WT animals, which indicates that targeting of Hifla with Fabp-Cre effectively eliminates the majority of detectable HIF-1 $\alpha$ 
A

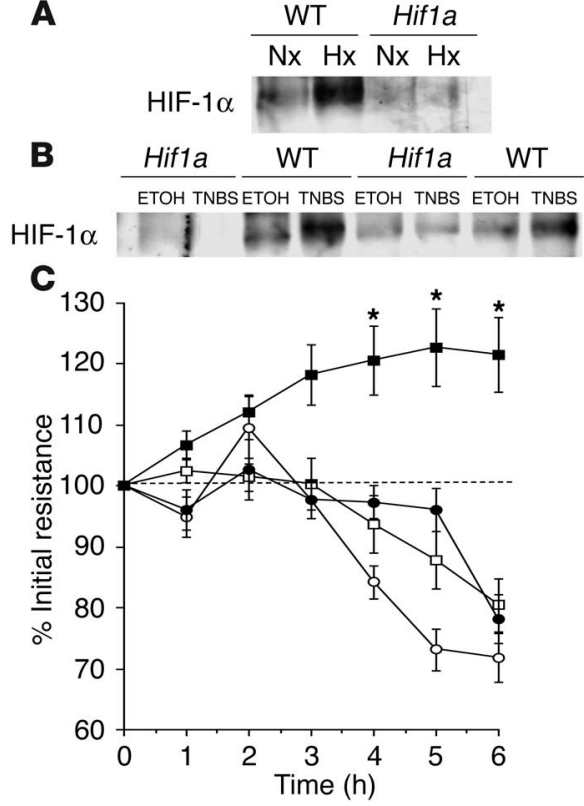

E
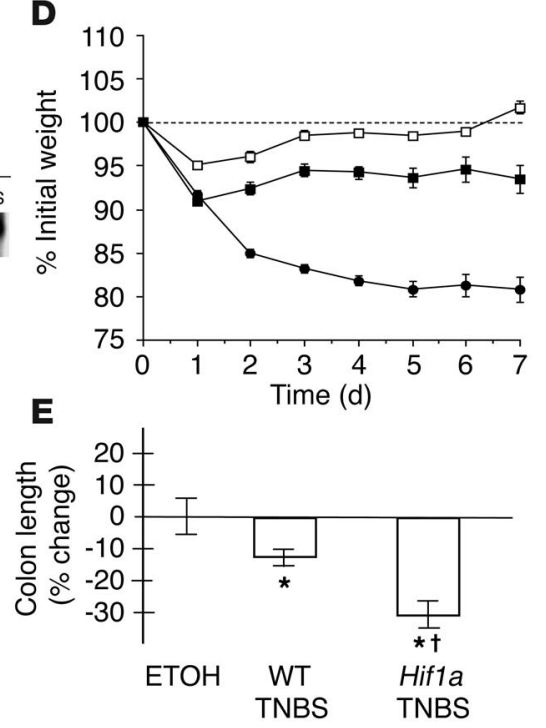

Figure 3

Impairment of barrier function through conditional deletion of Hif1a in intestinal epithelium. (A) Lack of HIF-1 induction in conditional Hif1a mutants in whole-body hypoxia. Western blot analysis of nuclear HIF-1 $\alpha$ levels from colonic scrapings of WT littermates or conditional Hif1a mice subjected to normoxia ( $\mathrm{Nx} ; 21 \% \mathrm{O}_{2}, 4 \mathrm{~h}$ ) or hypoxia $\left(\mathrm{Hx} ; 8 \% \mathrm{O}_{2}, 4 \mathrm{~h}\right.$ ). (B) Mucosal colonic scrapings 7 days after induction of TNBS colitis compared with vehicle control. HIF-1 $\alpha$ levels in Hif1 a mutants failed to respond, which indicates successful impairment of the HIF-1 pathway. (C) Ex vivo assay of barrier electrical resistance in colonic tissue explants. In samples derived from WT animals (filled squares), resistance increased in hypoxia $\left({ }^{\star} P \leq 0.05\right.$ after 3-6 h). In contrast, both in hypoxic samples from Hif1a conditional mutant animals (filled circles) and in all normoxic samples (WT, open squares; Hif1a mutant, open circles), measurements gradually dropped. (D) Changes of body weight following induction of TNBS colitis. When compared with their WT littermates (filled squares, $n=14$ ), Hif1a conditional mutant mice (filled circles, $n=25$ ) displayed a more severe clinical course with significantly greater and ongoing loss of weight $(P<0.01$ by ANOVA). No difference between vehicle control-treated Hif1a mutant and WT animals was observed (open squares). (E) Colon length relative to vehicle control-treated (ETOH) littermates 7 days after induction of colitis. Hif1a conditional mutant animals displayed significant shortening of the colon ${ }^{\dagger} P \leq 0.05$ vs. TNBS-treated WT, ${ }^{\star} P \leq 0.05$ vs. vehicle-treated controls).

induction. Since HIF-1 has been indirectly implicated in the protection of barrier function $(9,10)$, we determined whether functional barrier was influenced by Hifla deletion, using ex vivo electricalresistance measurements of colonic segments (Figure 3C). When subjected to hypoxia, colonic explants from WT littermate controls displayed a significant increase of electrical resistance as compared with specimens kept under atmospheric conditions $(P \leq 0.05)$. These findings are consistent with previous reports, which suggest that colonic epithelial hypoxia induces an innate adaptive phenotype that may render these cells more resistant to hypoxia than epithelial cells derived from other tissues (9). In contrast, colonic explants from conditional Hifla mutant animals showed an inability to mount the hypoxia-induced increase in epithelial resistance $(P<0.025$ by ANOVA). Genotype and experimental conditions did not affect tissue apoptosis or necrosis as determined by histological examination and lactate dehydrogenase (LDH) measurement (data not shown).

In parallel to the observed abnormalities of barrier function, TNBS colitis in Hifla conditional mutants was clinically more severe, leading to increased mortality (Hifla, 11/25, vs. WT, 3/14). Weight loss is a reliable method to assess TNBS colitis severity (28). Compared with littermate controls, conditional Hifla mutant animals lost weight more rapidly, to a greater extent, and failed to gain weight during the course of the 7-day experiment $(P \leq 0.05$ by ANOVA; Figure 3D). In addition, colon length (which shortens as a result of more severe inflammation) was significantly different in conditional Hifla mutants $(P \leq 0.05$ vs. littermate controls; Figure 3E). In contrast, histological examination using the scoring system of Elson et al. (29) insufficiently represented the observed clinical distinctions; this underlines the notion that significant functional disturbances can occur with no overt change in tissue architecture $(9,10)$.

For comparative purposes, we examined whether the course of colitis in models other than TNBS might be different between WT and conditional Hifla mutant animals. Here, we utilized oxazolone-induced colitis (30), a hapten-based model with a more Th2-type phenotype analogous to human ulcerative colitis. In keeping with our findings with TNBS, the course of oxazolone colitis in conditional Hif1a mutant animals was significantly worsened. For example, mortality was higher in the Hifla mutant animal population (10/18) compared with WT (2/13), body weight recovery was slower in Hifla mutant animals (body weights were $89.4 \% \pm 1.7 \%$ and $97.8 \% \pm 2.8 \%$ of initial weight at day 5 for Hifla mutant and WT animals, respectively; $P \leq 0.05$ ), and intestinal permeability to 4-kDa FITC-dextran was significantly increased compared with that in WT counterparts (3.4 \pm 0.44 -fold increase in Hifla mutant compared with WT animals; $P<0.01$ ). Taken together, these results indicate that the HIF-1 is an important barrier-protective mechanism for the severity of model colitis, including those induced by oxazolone and TNBS.

Mutant Vhlh mice express HIF-1 and ITF constitutively in the colon and are protected from bypoxia-induced permeability changes. Since we have previously shown that conditional inactivation of Vblh results in constitutively active HIF in the liver (25), we first investigated the effects of mutant Vblh targeted to the intestinal epithelium on colonic HIF-1 stability and hypoxia-induced loss of intestinal barrier function. For this, we subjected animals for 4 hours to normobaric hypoxia $\left(8 \% \mathrm{O}_{2}\right)$, a model we have used in the past to study epithelial barrier function in vivo $(9,10)$. As anticipated, tissue harvested from conditional Vhlh mutant animals manifested constitutive overexpression of both HIF-1 $\alpha$ and HIF- $2 \alpha$ (Figure 4, A and B). Littermate controls, in contrast, expressed normal HIF-1 $\alpha$ and HIF- $2 \alpha$ profiles, with low protein levels in normoxia and significant induction following hypoxic challenge. Based on previous work on HIF-dependent ITF expression and function (9), we also examined ITF regulation in these animals. Both ITF mRNA (Figure 4C) and protein levels (Figure 4D) were constitutively elevated in conditional Vhlb mutant mice. As hypoxia-inducible ITF levels correlate with intestinal barrier protection (9), we next examined intestinal permeability in ani- 


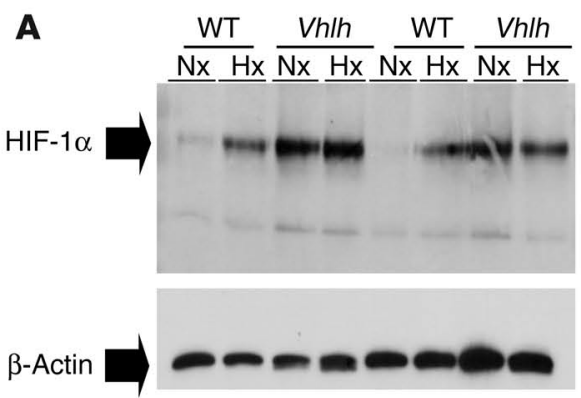

C

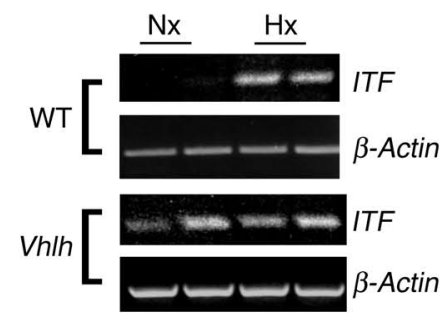

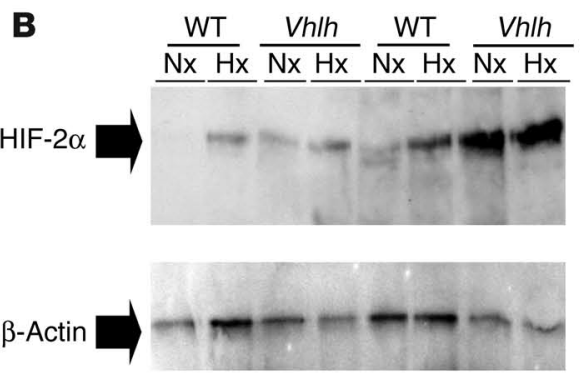

D

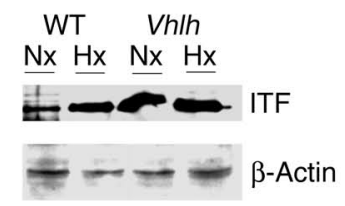

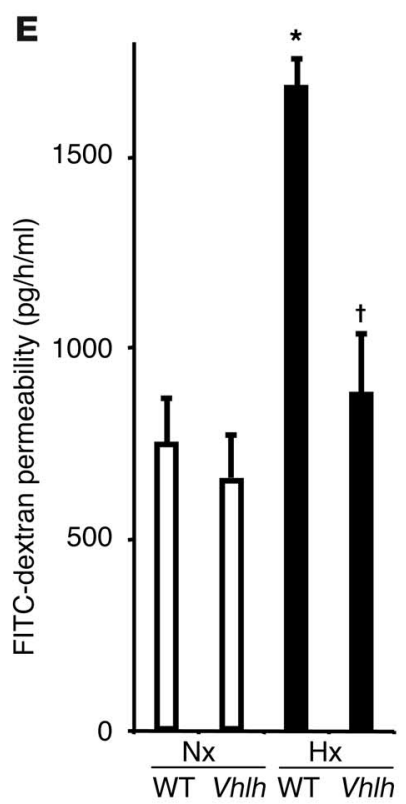

Figure 4

Conditional Vhlh deletion results in constitutive activation of the HIF-1 pathway in the whole-body hypoxia model. (A and B) Western blot analysis for HIF- $1 \alpha$ and HIF-2 $\alpha$ in conditional Vhlh mutant animals and WT littermates subjected to normoxia $\left(21 \% \mathrm{O}_{2}, 4\right.$ hours) or hypoxia (8\% $\mathrm{O}_{2}, 4$ hours). In contrast to WT animals, which display normal regulation, mutant animals constitutively overexpress HIF-1 $\alpha$ (A) and HIF-2 $\alpha$ (B). (C and D) HIF-1-dependent gene induction is exemplified by induction of ITF on both the transcriptional level (C) and the translational level (D) from colonic tissue derived from control and conditional Vhlh mutant animals subjected to normoxia or hypoxia. (E) Quantitation of serum FITC-dextran as a measure of intestinal permeability in control and conditional Vhlh mutant animals subjected to hypoxia or normoxia. Conditional deletion of Vhlh abrogated the increase in permeability observed in control animals $\left({ }^{\dagger} P<0.025\right.$ vs. hypoxic WT, ${ }^{*} P \leq 0.05$ vs. normoxic controls).

mals subjected to hypoxia. No differences in intestinal permeability to 4-kDa FITC-dextran were noted between littermate controls and conditional Vhlh mutant animals in normoxia (Figure 4E). In contrast, when exposed to hypoxia, conditional Vhlh mutant animals displayed a significantly lesser impairment of barrier function $(0.2 \pm 0.03$-fold increase; $P$ not significant vs. normoxia $)$ when compared with littermate controls $(2.3 \pm 0.3$-fold increase; $P<0.025)$. Such results strongly suggest that conditional Vhlb mutant mice are protected from intestinal hypoxia insult.

Vhlh mutant mice are protected from TNBS colitis and display an amplified HIF-1-dependent response. We next tested whether the conditional deletion of the Vhlh gene in the colonic epithelium would influence disease progression in TNBS colitis. In regard to weight loss, we observed a significant protection of the conditional mutants compared with littermate controls $(P<0.01$ by ANOVA; Figure $5 \mathrm{~A})$. Weight loss recovery in TNBS-exposed Vhlh mutants was indistinguishable from that in vehicle controls beyond 4 days of TNBS. In contrast, the weight loss profile of TNBS-treated littermate control animals remained significantly impaired over the entire 7-day observation period. Accordingly, colon length as an indicator of local disease severity was significantly lower in TNBS-treated animals compared with conditional Vhlh mutants after TNBS $(P \leq 0.05$; Figure $5 \mathrm{~B}$ ). The mortality was $0 / 12$ for Vblh mutants and $2 / 22$ for WT animals. Similar to our findings with the Hif1a mutant animals, histological examination yielded no significant distinction between treated Vhlh mutant animals and their WT littermates. At the gene transcriptional level, the initial findings of Figure $1 \mathrm{H}$ were supported by a significant overexpression of HIF-1 target genes in TNBS-treated WT animals relative to vehicle-treated littermate controls, including GLUT-1 $(1.7 \pm 0.3$-fold; $P \leq 0.05), I T F$ (2.0 \pm 0.6 -fold; $P \leq 0.05), M D R 1$ ( $2.9 \pm 1.6$-fold; $P \leq 0.05)$, and $C D 73$ (4.1 \pm 0.9 -fold; $P \leq 0.05$; Figure $5 C)$. In conditional Vhlb mutant animals, this mRNA induction was greatly enhanced for ITF (8.1 \pm 2.8 -fold; $P \leq 0.05), M D R 1$ (8.4 \pm 2.6 -fold; $P \leq 0.05)$, and $C D 73$ $(17.3 \pm 4.8$-fold; $P \leq 0.05)$. Other pathways of hypoxia inducibility did not seem to be affected in our model. Though responsive to TNBS treatment, differences in TNF- $\alpha$ levels were not statistically significant between TNBS-treated WT and Vhlb mutant animals (WT, $49 \pm 12.6$-fold TNF- $\alpha$ mRNA induction; Vhlh, $16 \pm 4.8$-fold TNF- $\alpha$ mRNA induction; $P \leq 0.05$ vs. control-treated littermates, $P=0.5$ within treatment populations). Consistent with our findings in hypoxia, significant intestinal epithelial barrier function in TNBS was afforded by overexpression of HIF in Vhlb mutant mice (Figure 5D; intestinal permeability rates: TNBS-treated WT, $3,298 \pm 680 \mathrm{pg} / \mathrm{h} / \mathrm{ml}$; TNBS-treated Vhlh mutants, $1,341 \pm 62$ $\mathrm{pg} / \mathrm{h} / \mathrm{ml}$; and vehicle-treated controls, $738 \pm 81 \mathrm{pg} / \mathrm{h} / \mathrm{ml} ; P \leq 0.05$ for WT vs. vehicle controls and vs. TNBS-treated $V h l b$ mutants). Taken together, these findings indicate that increased levels of epithelial HIF expression attenuate the severity of TNBS disease progression, at least in part through barrier-protective gene induction.

\section{Discussion}

The studies outlined here provide a molecular link between hypoxia and ongoing mucosal inflammation. Initial studies detailed the extent of hypoxia in the normal and inflamed intestine and revealed the epithelium as the major target for hypoxia. Utilizing conditional Hifla and Vblh mutant mice that constitutively express altered levels of epithelial HIF-1, we demonstrate a 

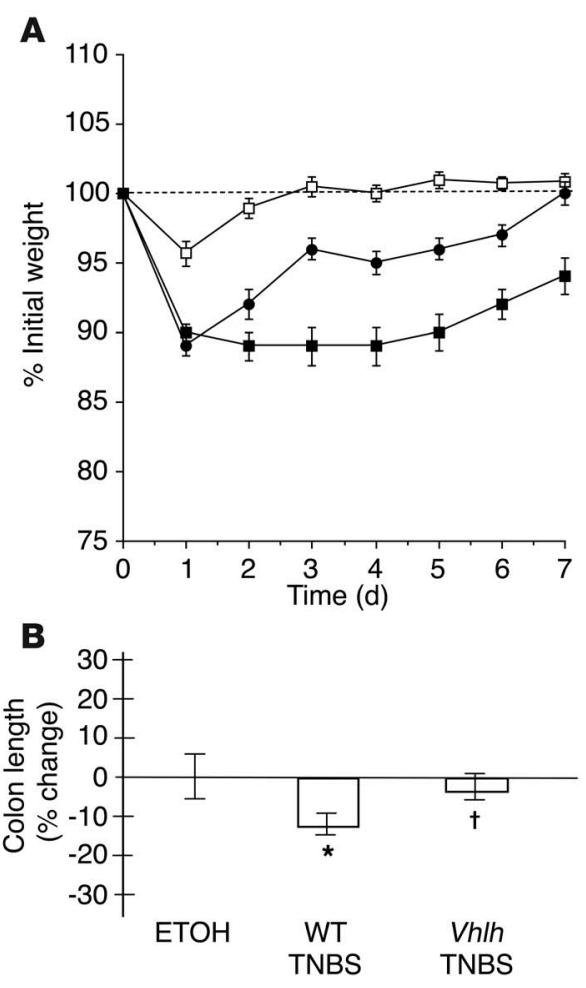
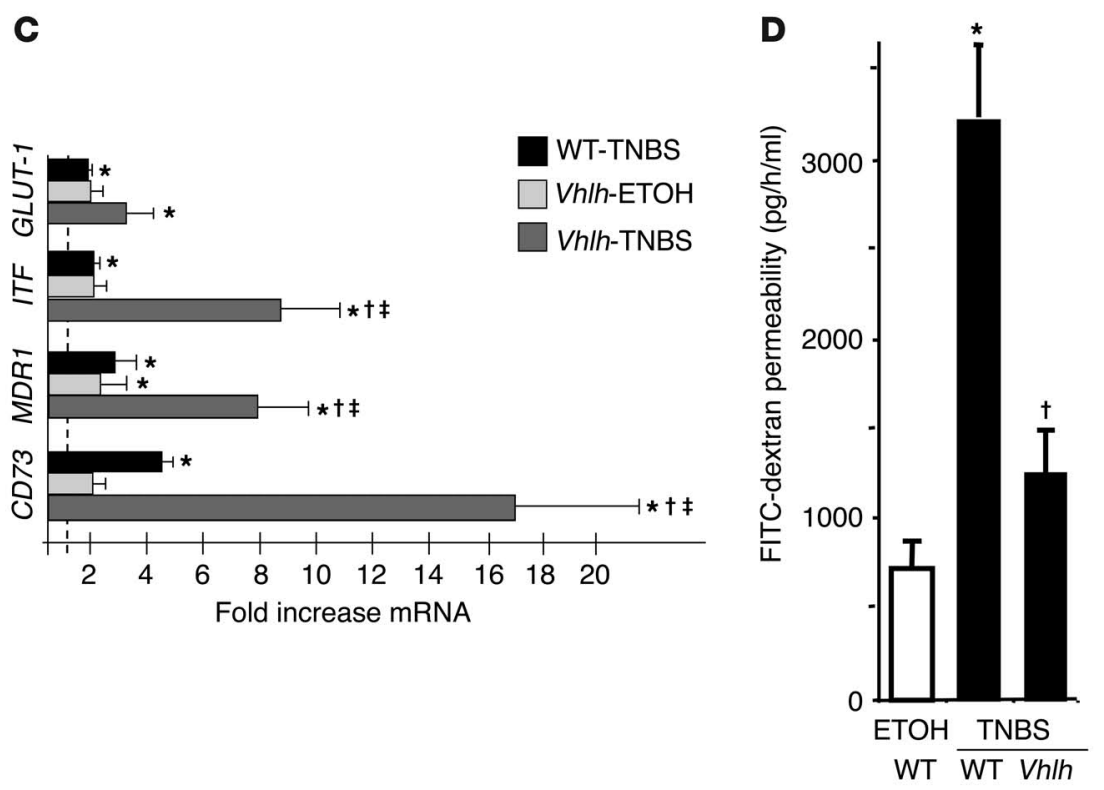

\section{Figure 5}

Conditional deletion of epithelial Vhlh protects from experimental colitis. (A) Vhlh mutant animals (filled circles, $n=12$ ) recovered their initial weight loss after administration of TNBS significantly faster than WT littermates (filled squares, $n=22 ; P<0.01$ by ANOVA). Values for vehicle control-treated Vhlh mutant and WT animals were combined (open squares). (B) Colonic length was significantly decreased in WT TNBS-treated animals compared with conditional Vhlh mutant animals with TNBS colitis ( ${ }^{\dagger} P \leq 0.05$ vs. TNBS-treated WT animals, ${ }^{*} P \leq 0.05$ vs. vehicletreated controls). (C) Real-time PCR analysis for GLUT-1, ITF, MDR1, and CD73. Data are presented as fold \pm SEM increase over vehicle control-treated WT animals (represented by the line at 1 -fold increase). While TNBS treatment significantly induced all 4 genes ( $P \leq 0.05$ vs. vehicle-treated WT), such differences were enhanced in conditional Vhlh mutant animals exposed to TNBS in regard to ITF, MDR1, and CD73 $\left({ }^{\dagger} P \leq 0.05\right.$ vs. TNBS-treated WT, $¥ P \leq 0.05$ vs. vehicle-treated Vhlh mutants). (D) Significantly lower flux of the FITC-dextran as a measure of intestinal permeability in TNBS-treated Vhlh mutant compared with TNBS-treated WT animals $\left({ }^{\dagger} P \leq 0.05 ; P\right.$ not significant for TNBS-treated Vhlh mutants vs. vehicle control-treated mice). As no difference was seen between mutant and WT vehicle control-treated animals (compare with Figure 4E), values were combined.

previously unappreciated innate protective mechanism in which HIF-1 ensures mucosal integrity through the induction of a number of barrier-protective genes.

A number of previous studies have suggested that the intestine is relatively hypoxic in the physiologic state $(20,31)$. Our findings with EF5 confirm this previous work and suggest that the healthy epithelium is likely to exist in a state of relative hypoxia. Given the anatomic locale of intestinal epithelia (e.g., steep oxygen gradient from the anaerobic lumen across the epithelium), it is interesting to speculate as to whether such "physiologic hypoxia" might subserve epithelial function and, as such, maintain expression of functional barrier-related proteins. Results from the ex vivo colonic explant model support this notion and suggest that relative hypoxia might promote barrier integrity. Additional support for this hypothesis was provided by previous work indicating that the barrier formed by intestine-derived epithelia may be uniquely resistant to hypoxic challenge (9). Elaboration on this observation led to the identification of barrier-protective gene products (e.g., ITF, P-GP, CD73), each of which has now been demonstrated to be transcriptionally regulated by HIF-1 (8-10). The loss of at least 2 of these molecules in vivo (ITF and P-GP) contributes significantly to the establishment of colitis in mice $(11,12)$. Moreover, an additional function of the intestinal epithelium is the absorption and transport of dietary iron (32). Recent studies suggest that at least 1 of the iron transporters, namely the transferrin receptor, is HIF-1 regulated (33). While it has been assumed that transferrin receptor induction by hypoxia provides a pathway for rapid increases in iron transport upon demand, it is also possible that such expression could contribute to basal iron transport in the intestine. Thus, additional work is necessary to determine whether lower oxygen tension in the intestine contributes to normal physiologic expression of functional epithelial genes.

It has long been hypothesized that hypoxia contributes to the inflammatory response (34), most likely through the combination of increased metabolic demands of inflamed tissue and altered blood flow as a result of tissue damage. In the present example of colitis, it was proposed that the vasculitis associated with such diseases as Crohn disease contributed significantly to the establishment and maintenance of chronic inflammation $(1,4)$. In addition, there is evidence for a complex system of bidirectional crosstalk between hypoxia and inflammation, since cytokines liberated during inflammation (e.g., IL-1, IFN- $\beta$, TNF- $\alpha$ ) may regu- 
late HIF-1 expression (35-37). Moreover, HIF-1 may either directly or indirectly influence expression of the chemokine IL-8 (38). It is interesting in this regard that TNF- $\alpha$ - which itself is known to be hypoxia-responsive, albeit through HIF-1-independent pathways $(39,40)$ - was not differentially expressed in colitic Vhlh-deficient animals. Our studies here with the TNBS colitis model reveal a potentially central role for HIF-1 as an endogenous protective pathway for inflammatory colitis and are consistent with a recent cDNA profiling study that suggested that HIF-1 $\alpha$ expression is induced in ulcerative colitis (41). As such, our work is paralleled by a number of publications that investigate HIF-1 function in a more complex inflammatory context such as respiratory distress syndrome (42), retinitis (43), arthritis $(36,44)$, and myeloid cell-mediated inflammation (24). In this context the distinction between inflammatory effector cells and target organ is noteworthy. HIF-1 seems to serve not as a general anti-inflammatory element, but rather as a factor that ensures the respective tissue function in the absence of adequate oxygen levels. This notion is supported by our observation that HIF-1 played a protective role in colonic epithelial cells attenuating clinical manifestations of colonic inflammatory disease, whereas increased expression of HIF-1 in myeloid cells led to a hyperinflammatory response in two models of acute inflammation (24).

While we have addressed the relative expression levels of a number of endogenously protective genes in TNBS colitis, we do not know the relative importance of these and likely other gene products to the protection afforded by epithelial HIF-1. HIF-dependent genes induced during colitis share at least 1 common mechanism, that of barrier support. Indeed, the initial studies demonstrating HIF-dependent expression of ITF and MDR1 were based on a transcriptional profile screening. This same screen also identified CD73 as a candidate protective molecule during hypoxia (10). Importantly, each of these molecules is largely expressed on the apical surface of mucosal epithelia, and each has been implicated, either directly or indirectly, in the regulation of barrier function. Thus, it would appear that at least 1 HIF-1-dependent pathway involves an "apical cluster" of genes in the protection of barrier in vivo. At present, we do not know whether the expression of these molecules is differentially influenced by HIF-1-independent mechanisms found within colitic tissues (e.g., inflammatory cytokines, reactive oxygen species, etc.). To this end, it was recently shown that conditional deletion of the $\beta$ chain (ARNT) of HIF by a keratinocyte-specific Cre-recombinase results in neonatal death due to skin barrier defects (45). These studies revealed that death was due to dehydration secondary to changes in skin ceramide compositions. It is noteworthy that, as we found to be the case in the colon, histological tissue architecture in Arnt-null epidermis was generally well preserved, supporting earlier observations $(9,10)$ that barrier integrity can be significantly compromised with no observable change in histology. Colonic barrier is provided by a single, undulating layer of columnar epithelia, while the dermal barrier is maintained by a multilayered cornified squamous epithelia. Although the colon and skin have vastly different morphologies, a number of common features relevant to general barrier properties should be considered. First, mucosal surfaces, including the skin and intestine, provide a physical barrier between biologic compartments, preventing the free mixing of antigenic material with the subepithelial lamina propria, which houses the mucosal immune system. Importantly in this regard, inappropriate responses to acute disruption of these bar- riers provide a major site for both infection and establishment of chronic inflammatory disease $(12,46)$. Second, mucosal epithelia are crucially positioned for electrolyte and fluid homeostasis. As with the case of Arnt-null mice, a loss of such barrier can result in severe fluid imbalances. While we did not notice gross changes in lumenal contents derived from Hifla-mutant animals, it is likely that compensatory mechanisms not found in the skin (e.g., mucin secretion) persist in the colon to compensate for frank fluid loss. Third, a remarkable feature of many barrier cell types, including the skin and intestine, is their potential for proliferation. Rapid turnover and differentiation from stem cell populations require high metabolic loads within these tissues, and, given the role of HIF-1 in coordination of numerous metabolic processes (47), it is not surprising that mucosal epithelia are significantly impacted by changes in functional HIF-1 expression.

Taken together, these results suggest that epithelial HIF-1 is a protective element in inflammatory mucosal diseases, particularly those that influence epithelial barrier function as a pathophysiologic manifestation. Efforts to better understand methods to harness HIF-1 as a therapeutic modality could prove beneficial for such diseases.

\section{Methods}

Generation of mice with inactivation of Hifla or Vhlb in the colon. Mice used here were of mixed genetic backgrounds (129, BALB/c, and FVB/N). For the production of conditional colon mutants, $\mathrm{Fabpl}^{4 \times \mathrm{at}-132} / \mathrm{Cre}$ mice (22) were bred to mice that carried either the Hif1a or the Vhlb 2-lox allele $(25,48)$. Genotyping of mice was carried out as described previously $(25,48)$. Conditional mutant mice (Hif1 $a^{2 l o x / 2 l o x} ; \mathrm{Fabpl}^{14 \times a t-132} / \mathrm{Cre}$ or Vhlh ${ }^{2 l o x} / 2 l o x ; \mathrm{Fabpl}^{4 \times \mathrm{at}-132} /$ Cre) and control mice ( $H i f 1 a^{2 l o x / 2 l o x}$ or Vhlb2lox/2lox $)$ were analyzed at 8-16 weeks of age. In order to determine the efficiency of colonic, Cre-mediated recombination for both conditional mutants, we isolated epithelial cells as described previously (49) and performed quantitative PCR from genomic DNA using primers spanning the targeted regions of the Hifla and Vhlb conditional alleles and primers detecting an undeleted control gene. Furthermore, we assessed the efficiency of Fabp-Cre-mediated recombination by immunohistochemical staining for Cre expression in colonic tissue from $V h l b$ mutant mice using a polyclonal anti-Cre antibody (Novagen), as well as in a lacZ reporter transgenic animal (WT for Vhlb) using X-gal staining as described previously (22). The following primers and probes were applied: Hifla forward, 5'-AGCAGGAGACTACAATACTTTCTTT3'; Hifla reverse, 5'-GCACAA CCAACAGGACTACAT-3'; Hif1a probe, 5'-(6FAM)-TGGACACCAGCACCCACATCAGG-(BHQ-1)-3'; Vhlb forward, 5'-CCAGACTGATCTAGGGTGTGA-3'; Vhlb reverse, 5'-TGTTT GTTTCCTTTCCTTTTCTTTT-3'; Vhlh probe, 5'-(TET)-CCAGGACAGCCAGGATTACACGAAG-(BHQ-1)-3'. All procedures involving animals were performed according to NIH guidelines for use of live animals and were approved by the Institutional Animal Care and Use Committee at Brigham and Women's Hospital.

Colitis models. TNBS or oxazolone colitis was induced with a modification of the techniques of Morris et al. (50) and Boirivant et al. (30), respectively. Briefly, mice were sensitized by epicutaneous application of either 1\% TNBS (Sigma-Aldrich) or 3\% oxazolone (4-ethoxymethylene-2phenyl-2-oxazolin-5-one; Sigma-Aldrich) in 100\% ethanol on day -7, followed by intrarectal administration of $5 \mu \mathrm{l} / \mathrm{g}$ body weight of $2.5 \%$ TNBS solution or $150 \mu \mathrm{l}$ of $1 \%$ oxazolone solution in $50 \%$ ethanol on day +7 . Vehicle-control animals received a corresponding volume of $50 \%$ ethanol alone. Only animals that showed an initial response to treatment were included in the study; this response was defined as a $5 \%$ loss of weight after induction of colitis. This resulted in the exclusion of 2 of $38 \mathrm{WT}$ 
mice, 3 of 28 Hifla conditional mutants, and 14 of 26 Vhlh conditional mutants from analysis. Similarly, deaths occurring within 24 hours of rectal instillation of either oxazolone or TNBS were not included in calculation of the mortality. As a further parameter, colon length was determined by measurement of the distance from the most distal aspect of the cecum to the most terminal aspect of the rectum.

In vivo detection of tissue hypoxia. The 2-nitroimidazole compound EF5 was a generous gift from Cameron Koch (University of Pennsylvania, Philadelphia, Pennsylvania, USA). EF5 was prepared by dissolution in $0.9 \%$ saline to a final concentration of $10 \mathrm{mM}$. Four hours prior to tissue harvest, mice received EF5 via intravenous injection to a volume of $1 \%$ of body mass. Frozen tissues after in vivo exposure to EF5 were cut to $10-\mu \mathrm{m}$ sections and stained as described previously (51) with Cy3-conjugated anti-EF5 mAb ELK3-51 (75 $\mu \mathrm{g} / \mathrm{ml}$; also provided by Cameron Koch). To assess antibody specificity, corresponding sections were incubated with ELK3-51 admixed with $0.5 \mathrm{mM}$ EF5 ("competed stain"). Tissue autofluorescence was documented after incubation with antibody carrier alone (data not shown). Slides were counterstained with $3 \mu \mathrm{M}$ DAPI (Invitrogen Corp.).

Western blot analysis. Nuclear and cytoplasmic fractions of mucosal scrapings (to enrich for epithelial cells) were isolated according to the protocol of Zhang et al. (52). Samples were normalized for protein content and resolved by nonreducing SDS-PAGE as described previously (40). Antibodies used for Western blotting included rabbit polyclonal anti-GLUT-1 $(1 \mu \mathrm{g} / \mathrm{ml}$; Alpha Diagnostic International Inc.), mouse monoclonal antiP-GP ( $1 \mu \mathrm{g} / \mathrm{ml}$; Signet Pathology Systems Inc.), rabbit polyclonal anti-ITF $(2 \mu \mathrm{g} / \mathrm{ml}$; a kind gift from Dan Podolsky, Massachusetts General Hospital, Boston, Massachusetts, USA), monoclonal mouse anti-HIF-1 $\alpha(1 \mu \mathrm{g} / \mathrm{ml}$; Novus Biologicals Inc.), mouse monoclonal HIF- $2 \alpha(1 \mu \mathrm{g} / \mathrm{ml}$; Novus Biologicals Inc.), and goat polyclonal anti-actin (Santa Cruz Biotechnology Inc.). Blots were washed, and species-matched peroxidase-conjugated secondary antibody was added. Labeled bands from washed blots were detected by enhanced chemiluminescence (Amersham Pharmacia Biotech).

Ex vivo barrier-resistance measurement. For determination of colonic resistance, the colon was harvested under sterile conditions, washed in PBS, and 3 samples per mouse and per condition were mounted in a snap well chamber as described previously (53). Before the first measurement, 30 minutes were allowed for equilibration of the tissue. For hypoxic exposure, samples were mounted and then transferred to a humidified hypoxic cell chamber (Coy Laboratory Products Inc.), where the medium was exchanged with preequilibrated hypoxic medium. Standard hypoxic conditions were $\mathrm{pO}_{2} 20$ torr, $\mathrm{pCO}_{2} 35$ torr, with the balance made up by nitrogen and water vapor. Electrical resistance was determined using a voltmeter (Evohm; World Precision Instruments Inc.). After completion of the 6-hour experimental time frame, the supernatant was assayed for lactate dehydrogenase (Promega Corp.), and the tissue was harvested for histological evaluation. Results were calculated as percent change compared with the respective starting measurement using samples from a total of 4 mice for each population.

Intestinal permeability in vivo. Intestinal permeability was examined using a FITC-labeled-dextran method, as described previously $(9,54)$. Briefly, mice were gavaged with $0.6 \mathrm{mg} / \mathrm{g}$ body weight of FITC-dextran (mol wt $4,000 \mathrm{kDa}$, at a concentration of $80 \mathrm{mg} / \mathrm{ml}$; Sigma-Aldrich) and exposed to normobaric hypoxia $\left(8 \% \mathrm{O}_{2}, 92 \% \mathrm{~N}_{2}\right)$ or room air for 4 hours $(n=4-6$ per condition). Following subjection to hypoxia or normoxia, cardiac puncture was performed and serum analysis of FITC concentration performed.

Transcriptional analysis. For transcriptional analysis, mucosal scrapings were homogenized in TRIzol (Invitrogen Corp.) using a 550 sonic dismembrator (Fisher Scientific International Co.) and RNA was isolated by phenol-chloroform extraction. After DNase digestion (GenHunter Corp.), reverse transcription was done using the Omniscript RT kit (QIAGEN Inc.) and oligo-dT primers (Invitrogen Corp.). Amplification was performed on an iCycler iQ real-time PCR detection system (Bio-Rad Laboratories Inc.) using the following murine gene-specific primers: ITF forward, 5'-GTAACAACCGTGGCTGCTG-3'; ITF reverse, 5'-GGCCCCCAGAACACAGTTATG-3'; GLUT-1 forward, 5'-CCTATGGCCAAGGACACA-3'; GLUT-1 reverse, 5'-CTGGTCTCAGGCAAGGAAAG-3'; CD73 forward, 5'-CTGGGGCACTCTGGTTTTGA; CD73 reverse, 5'-TCCCCGCAGGCACTTCTTTG; TNF- $\alpha$ forward, $5^{\prime}$-GCCTATGTCTCAGCCTCTTCTC; TNF- $\alpha$ reverse, 5 -ATCACCCCGAAGTTCAGTAGAC; $\beta$-Actin forward, 5'-AACCCTAAGGCCAACCGTGAA-3'; $\beta$-Actin reverse, $5^{\prime}$-TCACGCACGATTTCCCTCTCA-3'; MDR1 forward, 5'-GAGCTGTGACTATCTGAGGTGCT-3'; MDR1 reverse, 5'-GGCTTCTCATCTTGATGGAGTTA-3' . Cycle parameters were 2 minutes at $50^{\circ} \mathrm{C}, 1$ minute at $95^{\circ} \mathrm{C}$, then 40 cycles with 50 seconds at $95^{\circ} \mathrm{C}, 50$ seconds at $58^{\circ} \mathrm{C}$, and 50 seconds at $72^{\circ} \mathrm{C}$, followed by repetitive melting cycles to establish product specificity. Comparison of gene expression in a semiquantitative manner was performed based on the mathematical model of Pfaffl (55).

Data analysis. Weight-chart comparisons of data among each experimental group were carried out with ANOVA. Results from semiquantitative PCR were compared using Student's $t$ test. Values are expressed as means \pm SEM. $P$ values less than 0.05 were considered significant.

\section{Acknowledgments}

We thank Jeff Gordon (Washington University, St. Louis, Missouri, USA) for the generous gift of Fabpl ${ }^{4 x}$ at-132/Cre transgenic mice and Roderick Bronson for help with animal histology. We extend a special appreciation to Rudolf Jaenisch (Massachusetts Institute of Technology, Cambridge, Massachusetts, USA) for his support of the initial pilot studies. This work was supported by NIH grants DK50189 and HL60569 (to S.P. Colgan), DK02668 and DK062060 (to V.H. Haase), and PO1 DE13499, and by a grant from the Crohn's and Colitis Foundation of America (to J. Karhausen).

Received for publication January 15, 2004, and accepted in revised form August 31, 2004.

Address correspondence to: Volker H. Haase, University of Pennsylvania School of Medicine, Department of Medicine, 700 Clinical Research Building, 415 Curie Boulevard, Philadelphia, Pennsylvania 19104-6144, USA. Phone:(215) 573-1830; Fax:(215) 898-0189; E-mail: vhaase@mail.med.upenn.edu. Or to: Sean P. Colgan, Brigham and Women's Hospital, Center for Experimental Therapeutics and Reperfusion Injury, 20 Shattuck Street, 704 Thorn Building, Boston, Massachusetts 02115, USA. Phone: (617) 278-0599; Fax: (617) 278-6957; E-mail: colgan@zeus.bwh.harvard.edu.

J. Karhausen's present address is: Abteilung Anästhesiologie und Transfusionsmedizin, Universitätsklinik Tübingen, Tübingen, Germany.

\footnotetext{
1. Thornton, M., and Solomon, M.J. 2002. Crohn's disease: in defense of a microvascular aetiology. Int. J. Colorectal Dis. 17:287-297.

2. Hatoum, O.A., Binion, D.G., Otterson, M.F., and Gutterman, D.D. 2003. Acquired microvascular dysfunction in inflammatory bowel disease: loss of nitric oxide-mediated vasodilation. Gastroenter-

ology. 125:58-69.

3. Sands, B.E. 2004. From symptom to diagnosis: clinical distinctions among various forms of intestinal inflammation. Gastroenterology. 126:1518-1532.

4. Wakefield, A.J., et al. 1989. Pathogenesis of Crohn's disease: multifocal gastrointestinal infarction. Lancet. 2:1057-1062.
}

5. Yokoyama, K., et al. 2001. Obliterative arteritis with nitric oxide synthase and HLA-DR expression in Crohn's colitis. Hepatogastroenterology. 48:401-407.

6. Kruschewski, M., Foitzik, T., Perez-Canto, A., Hubotter, A., and Buhr, H.J. 2001. Changes of colonic mucosal microcirculation and histology in two colitis models. Dig. Dis. Sci. 46:2336-2343. 
7. Hauser, C.J., Locke, R.R., Kao, H.W., Patterson, J., and Zipser, R.D. 1988. Visceral surface oxygen tension in experimental colitis in the rabbit. J. Lab. Clin. Med. 112:68-71.

8. Comerford, K.M., et al. 2002. Hypoxia-inducible factor-1-dependent regulation of the multidrug resistance (MDR1) gene. Cancer Res. 62:3387-3394.

9. Furuta, G.T., et al. 2001. Hypoxia-inducible factor 1-dependent induction of intestinal trefoil factor protects barrier function during hypoxia. J. Exp. Med. 193:1027-1034.

10. Synnestvedt, K., et al. 2002. Ecto-5'-nucleotidase (CD73) regulation by hypoxia-inducible factor-1 (HIF-1) mediates permeability changes in intestinal epithelia. J. Clin. Invest. 110:993-1002. doi:10.1172/ JCI200215337.

11. Panwala, C.M., Jones, J.C., and Viney, J.L. 1998. A novel model of inflammatory bowel disease: mice deficient for the multiple drug resistance gene, mdr1a, spontaneously develop colitis. J. Immunol. 161:5733-5744.

12. Mashimo, H., Wu, D.C., Podolsky, D.K., and Fishman, M.C. 1996. Impaired defense of intestinal mucosa in mice lacking intestinal trefoil factor. Science. 274:262-265.

13. Semenza, G.L. 2000. HIF-1 and human disease: one highly involved factor. Genes Dev. 14:1983-1991.

14. Semenza, G.L. 2001. HIF-1 and mechanisms of hypoxia sensing. Curr. Opin. Cell Biol. 13:167-171.

15. Maxwell, P.H., et al. 1999. The tumour suppressor protein VHL targets hypoxia-inducible factors for oxygen-dependent proteolysis. Nature. 399:271-275.

16. Tanimoto, K., Makino, Y., Pereira, T., and Poellinger, L. 2000. Mechanism of regulation of the hypoxiainducible factor-1alpha by the von Hippel-Lindau tumor suppressor protein. EMBO J. 19:4298-4309.

17. Lando, D., Peet, D.J., Whelan, D.A., Gorman, J.J., and Murray, L.W. 2002. Asparagine hydroxylation of the HIF transactivation domain: a hypoxic switch. Science. 295:858-861.

18. Wakefield, A.J., et al. 1991. Granulomatous vasculitis in Crohn's disease. Gastroenterology. 100:1279-1287.

19. Evans, S.M., et al. 2000. Detection of hypoxia in human squamous cell carcinoma by EF5 binding. Cancer Res. 60:2018-2024.

20. Taylor, C.T., and Colgan, S.P. 1999. Therapeutic targets for hypoxia-elicited pathways. Pharm. Res. 16:1498-1505.

21. Semenza, G. 1999. Regulation of mammalian O2 homeostasis by hypoxia-inducible factor 1 . Annu. Rev. Cell Dev. Biol. 15:551-578.

22. Saam, J.R., and Gordon, J.I. 1999. Inducible gene knockouts in the small intestinal and colonic epithelium. J. Biol. Chem. 274:38071-38082.

23. Soriano, P. 1999. Generalized lacZ expression with the ROSA26 Cre reporter strain. Nat. Genet. 21:70-71.

24. Cramer, T., et al. 2003. HIF-1 is essential for myeloid cell-mediated inflammation. Cell. 112:645-657.

25. Haase, V.H., Glickman, J.N., Socolovsky, M., and Jaenisch, R. 2001. Vascular tumors in livers with targeted inactivation of the von Hippel-Lindau tumor suppressor. Proc. Natl. Acad. Sci. U. S. A
98: $1583-1588$.

26. Lee, J.Y., et al. 1998. Loss of heterozygosity and somatic mutations of the VHL tumor suppressor gene in sporadic cerebellar hemangioblastomas. Cancer Res. 58:504-508.

27. Vortmeyer, A.O., et al. 1997. von Hippel-Lindau gene deletion detected in the stromal cell component of a cerebellar hemangioblastoma associated with von Hippel-Lindau disease. Hum. Pathol. 28:540-543.

28. Neurath, M., Fuss, I., and Strober, W. 2000. TNBScolitis. Int. Rev. Immunol. 19:51-62.

29. Elson, C.O., et al. 1996. Hapten-induced model of murine inflammatory bowel disease: mucosa immune responses and protection by tolerance. J. Immunol. 157:2174-2185

30. Boirivant, M., Fuss, I.J., Chu, A., and Strober, W. 1998. Oxazolone colitis: a murine model of $\mathrm{T}$ helper cell type 2 colitis treatable with antibodies to interleukin 4. J. Exp. Med. 188:1929-1939.

31. Suski, M.D., Zabel, D., Levin, V., Scheuenstuhl, H., and Hunt, T.K. 1997. Effect of hypoxic hypoxia on transmural gut and subcutaneous tissue oxygen tension. In Oxygen transport to tissue XVIII. E.M. Nemoto and J.C. LaManna, editors. Kluwer Academic/Plenum Publishers. New York, New York, USA. 319-322.

32. Conrad, M.E., and Umbreit, J.N. 2000. Iron absorption and transport: an update. Am. J. Hematol. 64:287-298.

33. Tacchini, L., Bianchi, L., Bernelli-Zazzera, A., and Cairo, G. 1999. Transferrin receptor induction by hypoxia. HIF-1-mediated transcriptional activation and cell-specific post-transcriptional regulation. J. Biol. Chem. 274:24142-24146.

34. Ahlqvist, J. 1984. A hypothesis on the pathogenesis of rheumatoid and other non-specific synovitides. IV A. The possible intermediate role of local hypoxia and metabolic alterations. Med. Hypotheses. 13:257-302.

35. Der, S.D., Zhou, A., Williams, B.R., and Silverman, R.H. 1998. Identification of genes differentially regulated by interferon alpha, beta, or gamma using oligonucleotide arrays. Proc. Natl. Acad. Sci. U. S. A. 95:15623-15628.

36. Thornton, R.D., et al. 2000. Interleukin 1 induces hypoxia-inducible factor 1 in human gingival and synovial fibroblasts. Biochem. J. 350:307-312.

37. Albina, J.E., et al. 2001. HIF-1 expression in healing wounds: HIF-1alpha induction in primary inflammatory cells by TNF-alpha. Am. J. Physiol. Cell Physiol. 281:C1971-C1977.

38. Lee, T.H., Avraham, H., Lee, S.H., and Avraham, S. 2002. Vascular endothelial growth factor modulates neutrophil transendothelial migration via up-regulation of interleukin-8 in human brain microvascular endothelial cells. J. Biol. Chem. 277:10445-10451.

39. Taylor, C.T., Dzus, A.L., and Colgan, C.T. 1998. Autocrine regulation of epithelial permeability by hypoxia: role for polarized release of tumor necrosis factor $\alpha$. Gastroenterology. 114:657-668.

40. Taylor, C.T., Fueki, N., Agah, A., Hershberg, R.M., and Colgan, S.P. 1999. Critical role of cAMP response element binding protein expression in hypoxia-elicited induction of epithelial tumor necrosis factor- $\alpha$. J. Biol. Chem. 274:19447-19454.

41. Lawrance, I.C., Fiocchi, C., and Chakravarti, S. 2001. Ulcerative colitis and Crohn's disease: distinctive gene expression profiles and novel susceptibility candidate genes. Hum. Mol. Genet. 10:445-456.

42. Compernolle, V., et al. 2002. Loss of HIF-2alpha and inhibition of VEGF impair fetal lung maturation, whereas treatment with VEGF prevents fatal respiratory distress in premature mice. Nat. Med. 8:702-710.

43. Grimm, C., et al. 2002. HIF-1-induced erythropoietin in the hypoxic retina protects against light-induced retinal degeneration. Nat. Med. 8:718-724.

44. Hollander, A.P., Corke, K.P., Freemont, A.J., and Lewis, C.E. 2001. Expression of hypoxia-inducible factor 1alpha by macrophages in the rheumatoid synovium: implications for targeting of therapeutic genes to the inflamed joint. Arthritis Rheum. 44:1540-1544.

45. Takagi, S., et al. 2003. Alteration of the 4-sphingenine scaffolds of ceramides in keratinocytespecific Arnt-deficient mice affects skin barrier function. J. Clin. Invest. 112:1372-1382. doi:10.1172/JCI200318513.

46. Hermiston, M.L., and Gordon, J.I. 1995. Inflammatory bowel disease and adenomas in mice expressing a dominant negative $\mathrm{N}$-cadherin. Science. 270:1203-1207.

47. Iyer, N.V., et al. 1998. Cellular and developmental control of $\mathrm{O} 2$ homeostasis by hypoxia-inducible factor 1 alpha. Genes Dev. 12:149-162.

48. Ryan, H.E., Lo, J., and Johnson, R.S. 1998. HIF-1 alpha is required for solid tumor formation and embryonic vascularization. EMBOJ. 17:3005-3015.

49. Little, D., et al. 2003. PI3K signaling is required for prostaglandin-induced mucosal recovery in ischemia-injured porcine ileum. Am. J. Physiol. Gastrointest. Liver Physiol. 284:G46-G56.

50. Morris, G.P., et al. 1989. Hapten-induced model of chronic inflammation and ulceration in the rat colon. Gastroenterology. 96:795-803.

51. Laughlin, K.M., et al. 1996. Biodistribution of the nitroimidazole EF5 (2-[2-nitro- $1 H$-imidazol-1yl]$\mathrm{N}$-[2,2,3,3,3-pentafluoropropyl] acetamide) in mice bearing subcutaneous EMT6 tumors. J. Pharmacol. Exp. Ther. 277:1049-1057.

52. Zhang, X., et al. 1997. Isolation of nuclear proteins from human brain. Biotechniques. 22:849-850.

53. El Asmar, R., et al. 2002. Host-dependent zonulin secretion causes the impairment of the small intestine barrier function after bacterial exposure. Gastroenterology. 123:1607-1615

54. Napolitano, L.M., Koruda, M.J., Meyer, A.A., and Baker, C.C. 1996. The impact of femur fracture with associated soft tissue injury on immune function and intestinal permeability. Shock. 5:202-207.

55. Pfaffl, M.W. 2001. A new mathematical model for relative quantification in real-time RT-PCR. Nucleic Acids Res. 29:2002-2007. 